Short Research Communication

\title{
Parkinson's disease might increase the risk of cerebral ischemic lesions
}

\author{
In-Uk Song'ㄹ, Ji-Eun Lee², Do-Young Kwon³ ${ }^{3}$ Jeong-Ho Park ${ }^{4}$, Hyeo-Il Ma ${ }^{5 凶}$ \\ 1. Department of Neurology, Incheon St. Mary's Hospital, The Catholic University of Korea, Seoul, South Korea; \\ 2. Department of Neurology, National Health, Insurance Corporation Ilsan Hospital, Ilsan, South Korea; \\ 3. Department of Neurology, Korea University Ansan Hospital, Korea University, Ansan, South Korea; \\ 4. Department of Neurology, College of Medicine, Soonchunhyang University, Seoul, South Korea; \\ 5. Department of Neurology, College of Medicine, Hallym University, Anyang, South Korea. \\ $\triangle$ Corresponding author: Hyeo-Il Ma, MD. Department of Neurology, College of Medicine, Hallym University, 96 Pyungchon-dong, Anyang-si, Gyeonggi-do, \\ 431-796, Korea. Tel: +82-31-380-3740; E-mail: hima@hallym.ac.kr. \\ (C) Ivyspring International Publisher. This is an open access article distributed under the terms of the Creative Commons Attribution (CC BY-NC) license \\ (https://creativecommons.org/licenses/by-nc/4.0/). See http://ivyspring.com/terms for full terms and conditions.
}

Received: 2016.10.21; Accepted: 2017.01.14; Published: 2017.03.11

\begin{abstract}
Background: Parkinson's disease (PD) is the second most common neurodegenerative disease in the elderly. Cerebrovascular diseases such as cerebral ischemic lesion (CIL) also commonly occur in elderly adults. However, previous studies on the relationship between PD and cerebrovascular disease have not found consistent results. Therefore, we conducted this study to evaluate whether or not PD is related to an increased prevalence of ischemic cerebrovascular lesions.

Methods: This study recruited 241 patients with PD and 112 healthy controls (HCs). All subjects underwent brain magnetic resonance imaging and general neuropsychological tests. The motor severity of PD was evaluated according to the Hoehn and Yahr stage (HY stage), and the severity of CIL in all subjects was classified according to Fazekas grade. The PD patients were classified into two subgroups according to HY stage (Group 1 - HY 1, 2; Group 2 - HY 3 to 5).

Results: Among all PD patients, $76 \%$ had small vessel disease, while $44 \%$ of all HCs had small vessel disease $(p<0.001)$. Regarding the difference between the two subgroups according to motor severity, group 2 showed significantly higher Fazekas scale score and more severe CIL, indicating a higher prevalence of small vessel disease compared to group 1.

Conclusion: This study demonstrates that PD patients have a significantly higher prevalence of CIL compared to HCs. Therefore, although the present study is not a large-scale study, we cautiously suggest that PD can play an important role as a risk factor in the occurrence of ischemic cerebrovascular disease.
\end{abstract}

Key words: Parkinson's disease; cerebral ischemic lesion; Fazekas scale

\section{Introduction}

It is well-known that Parkinson's disease (PD) is the second most common neurodegenerative disease in the elderly; however, the etiology of PD remains unclear. Recently, the role of concurrent medical problems has been a major concern surrounding PD. Cerebrovascular disease such as cerebral ischemic lesion (CIL) is the most common medical issue in the elderly[1]. However, previous epidemiological and clinico-pathological studies on the relationship between PD and cerebrovascular disease have found inconsistent results[2]. Stroke related mortality was determined to be 1.5 and 3.6 times higher in PD patients based on some previous studies, while other studies reported difference in stroke-related mortality between patients with PD and the general population[3,4]. In a case-control study of $200 \mathrm{PD}$ patients, Struck et al. reported a reduced cumulative risk of ischemic stroke in the PD population, probably due to less severe atherosclerosis related to lower tobacco use[5]. Korten et al. also found a lower than expected frequency of PD cases among stroke patients in the Maastricht Stroke Registry and speculated that 
dopamine deficiency may have a protective effect against stroke[6]. However, a retrospective case-control study of 119 PD patients and age-matched controls found no differences in the cumulative incidence of ischemic stroke, hypertension, and diabetes mellitus between both groups and failed to demonstrate protection from stroke in PD patients [7]. Furthermore, a recent population-based, propensity score-matched, longitudinal follow-up study showed an increased risk of ischemic stroke after diagnosis of PD[2]. Despite the inconsistent findings from previous studies, there have been few studies clarifying the relationship between $\mathrm{PD}$ and ischemic stroke. Therefore, we conducted this study to evaluate whether or not PD is related to an increase in the prevalence of ischemic cerebrovascular lesions and to determine if there is an increased risk of ischemic cerebrovascular lesions in PD patients.

\section{Methods}

This study was approved by the local ethics committee, and each participant provided written informed consent. Subjects were recruited between January 2007 and January 2016 at the outpatient movement disorder clinic of multiple medical centers. All consecutive patients and healthy subjects underwent brain magnetic resonance imaging (MRI) to evaluate the extent of cerebral ischemic lesion (CIL) and to exclude the presence of other brain lesions. In addition, an experienced radiologist and a neurologist, who were both blinded to the clinical status of all subjects, assessed the brain MRIs until consensus on the presence of CIL was achieved. Evaluation procedures consisted of a detailed medical history, physical and neurological examination, and neuropsychological assessment using the Mini-Mental State Examination (MMSE), the extended version of the Clinical Dementia Scale (CDR) with the sum of the box score of the CDR (SOB) and Global Deterioration Scale (GDS). All PD patients were diagnosed according to the United Kingdom Parkinson's Disease Society Brain Bank Clinical Diagnosis Criteria for Parkinson's Disease. Motor severity of PD patients was evaluated according to the Hoehn and Yahr stage (HY stage). Severity of CIL in all subjects was evaluated by white matter changes induced by ischemic lesions, which were classified according to Fazekas grade[8]. In addition, patients in the PD group were classified into two subgroups to evaluate the severity of ischemic white matter lesions according to motor severity of PD patients. The subgroups were defined as follows: group 1, patients with HY stage 1 or 2; group 2, patients with HY stage 3,4 , or 5 . The healthy control (HC) group was matched based on age, gender, and education level to patients in the PD group. The HCs did not have any history or symptoms of PD, memory impairment, or other cognitive dysfunctions, and they did not have a history of other neurological diseases such as head trauma, epilepsy, or stroke or brain surgery or medical diseases. The presence of hypertension, diabetes mellitus, hypercholesterolemia, and cigarette smoking were also assessed by evaluating medical histories and laboratory findings since these risk factors can affect the occurrence of ischemic white matter lesions. Therefore, we excluded all subjects with the above-mentioned risk factors from this study. Hypertension was defined as systolic blood pressure $\geq 140 \mathrm{~mm} \mathrm{Hg}$, diastolic blood pressure $\leq 90$ $\mathrm{mm} \mathrm{Hg}$, and/or the current use of antihypertensive medications. Diabetes mellitus (DM) was defined as a history of fasting glucose level $\geq 110 \mathrm{mg} / \mathrm{dl}$ or the current use of hypoglycemic agents. Hypercholesterolemia was defined as total cholesterol concentration $\geq 220 \mathrm{mg} / \mathrm{dl}$ or the current use of lipid-lowering agents. Cigarette smoking was defined as present if the patient reported smoking cigarettes at least once during the past five years. All statistical analyses were performed using the SPSS software version 18.0 package. The independent T-test was used for the comparison of continuous variables, and Pearson's Chi-square analyses were used for the comparison of categorical variables. Values are expressed as means and standard deviations. Statistical significance was assumed at a false detection rate less than $5 \%$.

\section{Results}

The demographic characteristics of the PD patient and HC groups are summarized in Table 1. A total of 353 subjects were recruited in this study. Among these subjects, we identified 241 patients with PD and 112 HCs. There were no overall significant differences in age or gender distribution between the PD patients and HCs. PD patients showed significantly lower MMSE scores and higher Fazekas scale scores compared to HCs. Among all PD patients, $76 \%$ had small vessel disease, while $44 \%$ of HCs had small vessel disease. Namely, PD patients demonstrated a significantly higher prevalence of small vessel disease compared with HCs $(p<0.001)$.

Regarding the difference between the two subgroups according to motor severity, we classified 162 of the PD patients in group 1 and 80 of the PD patients in group 2. The two groups showed no significant differences in gender. However, when comparing the age between the two groups, group 2 had a higher average age compared to group 1. Group 2 also showed significantly lower MMSE scores and 
higher CDR with SOB and GDS compared with group 1. Likewise, group 2 showed significantly higher Fazekas scale scores and more severe CIL indicating small vessel disease compared to group 1 (Table 2).

Table 1. Baseline Characteristics of the PD and Healthy Control Groups.

\begin{tabular}{llll}
\hline & PD & Healthy Control & $p$-value \\
\hline Number & 241 & 112 & - \\
Male* $^{*}$ & 151 & 90 & 0.639 \\
Age & $72.29 \pm 8.06$ & $71.21 \pm 8.74$ & 0.269 \\
MMSE & $20.37 \pm 6.28$ & $26.54 \pm 2.45$ & $<0.001$ \\
Fazekas Scale & $1.23 \pm 0.83$ & $0.60 \pm 0.75$ & $<0.001$ \\
Small vessel disease $^{*}$ & 182 & 49 & $<0.001$ \\
\hline
\end{tabular}

*Value is number and calculated by Chi-square test.

PD: Parkinson's disease; MMSE: Mini-mental state examination.

Table 2. Comparison between two groups of PD Group.

\begin{tabular}{|c|c|c|c|c|}
\hline & & Group 1 & Group 2 & $p$-value \\
\hline Number & & 162 & 80 & - \\
\hline Male $^{*}$ & & 62 & 28 & 0.672 \\
\hline Age & & $71.19 \pm 8.14$ & $74.51 \pm 7.43$ & 0.002 \\
\hline MMSE & & $21.69 \pm 5.96$ & $17.68 \pm 6.07$ & $<0.001$ \\
\hline CDR & & $0.57 \pm 0.41$ & $0.88 \pm 0.55$ & $<0.001$ \\
\hline Sum of Box of CDR & & $2.38 \pm 2.66$ & $5.18 \pm 4.31$ & $<0.001$ \\
\hline GDS & & $3.01 \pm 1.25$ & $4.08 \pm 1.09$ & $<0.001$ \\
\hline \multirow[t]{4}{*}{ Fazekas Scale* } & 0 & 35 & 4 & - \\
\hline & 1 & 88 & 43 & - \\
\hline & 2 & 28 & 22 & - \\
\hline & 3 & 11 & 11 & - \\
\hline Mean of Fazekas Scale & & $1.09 \pm 0.81$ & $1.50 \pm 0.80$ & $<0.001$ \\
\hline Small vessel disease* & & 114 & 68 & 0.017 \\
\hline
\end{tabular}

*Value is number and calculated by Chi-square test.

PD: Parkinson's disease; MMSE: Mini-mental state examination.

CDR: Clinical Dementia Rating; GDS: Global Deterioration Scale.

Group 1: Hoehn and Yahr scale(H-Y) 1 and 2; Group 2: $\mathrm{H}-\mathrm{Y} \geq 3$.

\section{Discussion}

There have been conflicting results from previous epidemiological and clinico-pathological studies on the relationship between cerebrovascular lesions and PD [5-8]. The present study showed that newly diagnosed PD was associated with an increase in silent small vessel diseases compared to HCs. Whereas many previous studies have focused on cerebrovascular diseases based on a history of stroke with neurological deficits during the lifetime of PD patients $[3,6,7]$, our study evaluated whether PD patients have a higher prevalence of silent ICLs. In contrast to the results from the present study, several previous studies have suggested a reduced risk of ischemic stroke in PD patients. These findings have been explained by the suggestion that dopamine deficiency has a protective effect against stroke, and that PD patients have lower tobacco use, which is a known risk factor of atherosclerosis[5,6]. However, previous postmortem studies neither indicated a significant increase or decrease in the prevalence of cerebrovascular lesions nor a greater susceptibility to death from stroke in the populations studied[4,8]. A study by Levine et al. also failed to demonstrate the protection of PD patients from stroke[7]. Furthermore, recent studies have reported a significantly increased risk of ischemic stroke in PD patients, although the underlying mechanism and reasons for this association are unclear[2,8]. Some studies have suggested that PD is mainly associated with certain vascular risk factors, such as diabetes and hypertension[2]. However, the present study excluded PD patients with ischemic stroke risk factors including diabetes, hypertension, and hyperlipidemia to investigate the risk of ICLs in PD. Therefore, based on the results of this study, we strongly assert that the occurrence of ICL is increased by only PD and not due to other vascular risk factors.

We suggest several possible reasons for the positive association between PD and ICL. First, supine hypertension and orthostatic hypotension can result from autonomic dysfunction in PD[9]. Orthostatic hypotension is among the most frequent and troublesome nonmotor symptoms of PD[10]. In one community cohort of PD patients who survived 20 years from diagnosis, $48 \%$ had symptomatic orthostatic hypotension[10]. Supine hypertension and orthostatic hypotension in PD could induce ischemic white matter damage. Therefore, supine hypertension and orthostatic hypotension have been suggested as risk factors of ischemic stroke[9]. Second, oxidative stress is one of the many possible pathogeneses of PD since it contributes to dopamine cell degeneration[11]. Furthermore, oxidative stress is considered to play an important role in endothelial dysfunction and the pathogenesis of atherosclerosis, which can increase the risk of cardiovascular and cerebrovascular events[2,12]. The link between PD and ischemic stroke can be attributed to a common pathogenesis pathway, namely oxidative stress, and the occurrence of PD might indicate higher cumulative oxidative stress, leading to a higher risk of ischemic stroke in PD patients[2]. Third, neuroinflammation potentially underlying PD might contribute to ICL in PD, since neuroinflammation itself is also a pathogenesis leading to vascular atherosclerosis [1].

The main limitation of the present study is that it is not a longitudinal follow-up study that followed patients over a long period of time. In addition, this study is limited by the relatively small sample size. Therefore, large-scale studies performed in multiple centers are needed to further clarify the association between PD and ICL. In this study, a definite diagnosis of PD was confirmed by neuropathological findings; however, we did not carry out any 
neuropathological investigation because the patients were still alive. Therefore, we cannot clearly differentiate typical PD from atypical Parkinsonism including Parkinson-plus syndrome. However, we attempted to reduce these confounders through detailed neurological examinations by two or more experts who are specialists in movement disorders including Parkinson's disease.

In summary, we found that PD patients without other stroke risk factors have a significantly higher prevalence of ischemic cerebrovascular lesions compared to healthy controls. Therefore, although the present study is not a large-scale study, we cautiously suggest that PD itself can play an important role as a risk factor of occurrence of ischemic cerebrovascular disease. Additionally, we emphasize that larger and longitudinal studies conducted in multiple centers are needed to further clarify the role of PD as an ischemic stroke risk factor.

\section{Competing Interests}

The authors have declared that no competing interest exists.

\section{References}

1. Song IU, Kim YD, Cho HJ, et al.: The effects of silent cerebral ischemic lesions on the prognosis of idiopathic parkinson's disease. Parkinsonism Relat Disord 2013;19:761-763.

2. Huang YP, Chen LS, Yen MF, et al.: Parkinson's disease is related to an increased risk of ischemic stroke-a population-based propensity score-matched follow-up study. PLoS One 2013;8:e68314.

3. Gorell JM, Johnson CC, Rybicki BA: Parkinson's disease and its comorbid disorders: An analysis of michigan mortality data, 1970 to 1990. Neurology 1994;44:1865-1868.

4. Mastaglia FL, Johnsen RD, Kakulas BA: Prevalence of stroke in parkinson's disease: A postmortem study. Mov Disord 2002;17:772-774.

5. Struck LK, Rodnitzky RL, Dobson JK: Stroke and its modification in parkinson's disease. Stroke 1990;21:1395-1399.

6. Korten A, Lodder J, Vreeling F, et al.: Stroke and idiopathic parkinson's disease: Does a shortage of dopamine offer protection against stroke? Mov Disord 2001;16:119-123.

7. Levine RL, Jones JC, Bee N: Stroke and parkinson's disease. Stroke 1992;23:839-842.

8. Becker C, Jick SS, Meier CR: Risk of stroke in patients with idiopathic parkinson disease. Parkinsonism Relat Disord 2010;16:31-35.

9. McDonald C, Newton JL, Burn DJ: Orthostatic hypotension and cognitive impairment in parkinson's disease: Causation or association? Mov Disord 2016;31:937-946.

10. Lim SY, Lang AE: The nonmotor symptoms of parkinson's disease--an overview. Mov Disord 2010;25 Suppl 1:S123-130.

11. Facecchia K, Fochesato LA, Ray SD, et al.: Oxidative toxicity in neurodegenerative diseases: Role of mitochondrial dysfunction and therapeutic strategies. J Toxicol 2011;2011:683728.

12. Harrison D, Griendling KK, Landmesser U, et al.: Role of oxidative stress in atherosclerosis. Am J Cardiol 2003;91:7A-11A. 